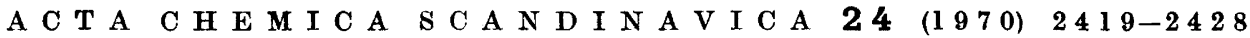

\title{
Relaxation Spectra and Kinetic Analysis of the H-Bond Association of Amides
}

\author{
JøRGEN RASSING and FRANK GARLAND \\ Chemistry Laboratory III, University of Copenhagen, Copenhagen, Denmark
}

\begin{abstract}
The hydrogen bond association of $N$-methyl acetamide dissolved in carbon tetrachloride has been investigated by means of ultrasonic absorption in the frequency range $10-300 \mathrm{Mc}$. The sound absorption shows a relaxation which is described by a single relaxation time. The concentration dependence of this time in the concentration range $0.02-0.1 \mathrm{M}$ is in agreement with the assumption that only a monomerdimer equilibrium exists. However, this model is contradicted by other types of experiments and it is shown that in this concentration range the data also agree with the generally accepted association model with several polymeric species present. The rate constants for this model are evaluated. At higher concentrations the values of the relaxation times differ from the values predicted from the polymerization model. This fact is attributed to a change in the thermodynamic properties of the solution.
\end{abstract}

$\mathrm{T}$ he structural character and the nature of the molecular association of amides are of particular interest because of the importance of amides in biological systems. As a result a variety of investigations of these systems have been carried out. Recently Rassing and Østerberg ${ }^{1}$ have investigated different mixtures of $N$-methyl acetamide (NMA) and $N, N$-dimethyl acetamide (DMA) by means of the ultrasonic absorption technique. The relaxation observed is suggested to be attributed to either an internal rotation about the $\mathrm{C}-\mathrm{N}$ bond or to the equilibrium between hydrogen bonded chains of NMA molecules. The former mechanism is rejected because it does not lead to numerical agreement with the experimental results, and consequently the latter mechanism is preferred. The fact that the relaxation frequency was found to be approximately independent of the concentration of NMA lead to an estimate of the common rate constant for depolymerization. A better check on the agreement between the polymerization model and the relaxational behavior of the system as well as a detailed kinetic analysis require a knowledge of the different equilibrium constants involved in the polymerization. ${ }^{2}$ This knowledge is not available for mixtures of NMA and DMA. 
The association mechanism and corresponding equilibrium constants for the hydrogen bond polymerization of NMA dissolved in carbon tetrachloride are known from infrared studies. ${ }^{-4}$ Consequently the possibility of a check and a detailed kinetic analysis as mentioned above exists for this system if the relaxational behavior were known.

In this paper we present the ultrasonic relaxation spectra of different solutions of NMA and NMA/DMA mixtures in carbon tetrachloride, and we discuss the extent to which agreement with the reported infrared results exists.

\section{THEORY}

The sound absorption caused by the relaxation of a single reaction is described by the following equation:

$$
\frac{\alpha}{\nu^{2}}=\frac{A}{1+(2 \pi \nu \tau)^{2}}+B
$$

where $\alpha$ is the sound absorption coefficient, $\nu$ is the frequency of the sound wave, $\tau$ is the relaxation time, and $A$ is the relaxation strength which is a function of the thermodynamics of the system and of the concentration of solute. $B$ is the background absorption, defined as the value of $\alpha / \nu^{2}$ measured at high enough frequencies for the absorption due to the chemical reaction to be negligible. If a multistep reaction is considered and thus several relaxation times are involved, the sound absorption coefficient can be expressed by

$$
\frac{\alpha}{\nu_{2}}=\sum_{i} \frac{A_{i}}{1+\left(2 \pi \tau_{i} \nu\right)^{2}}+B
$$

where the index $i$ refers to the $i:$ th step.

Even if experimental data fit eqn. (1) it does not necessarily mean, however, that the relaxation is caused by a single reaction step. If for a given multistep mechanism either

or

$$
\begin{gathered}
\tau_{1} \cong \tau_{2} \cong \tau_{3} \cong \ldots \cong \tau_{i} \\
A_{j} \ll \sum_{i \neq j} A_{i}
\end{gathered}
$$

then (2) becomes formally identical with eqn. (1) and the data caused by the multistep mechanism fit the one relaxation time equation. The consequences of (3) for a two step mechanism have been discussed recently, ${ }^{5}$ while (4) has been used in the interpretation of the relaxation spectra of benzyl alcohol dissolved in cyclohexane. ${ }^{6}$

The equilibrium between monomers and hydrogen bonded polymers of NMA molecules involves a large number of reaction steps. If only open chains are formed the simplest reaction scheme would be: 


$$
\begin{gathered}
N_{1}+N_{1} \rightleftharpoons N_{2} \\
N_{1}+N_{2} \rightleftharpoons N_{3} \\
\dot{\cdot} \\
\dot{\cdot} \\
N_{1}+N_{i} \rightleftharpoons N_{i+1}
\end{gathered}
$$

where $N_{i}$ denotes a polymer with $i$ molecules of NMA. This model has been applied to data obtained by equilibrium studies, where the interpretation is based on the assumption that the equilibrium constant for the dimerization step, $K_{1}$, is different from that of the subsequent steps, $K_{n}$; and that the latter may be taken as independent of $i{ }^{\text {s-4 }}$ In analogy with this the forward and reverse rate constants, $k_{i}$ and $k_{-i}$, of all but the first step are assumed to be independent of the degree of polymerization. The kinetics of the model is described by $n$ differential equations, where $n$ is the number of polymeric species considered. By introducing the concentration perturbations produced when sound is propagated through the system, by neglecting higher order terms in these perturbations, and by setting

$$
k_{-i}=k_{-n} \quad i \geq 1
$$

the following set of $n$ coupled differential equations is obtained:

$$
\dot{a}=-k_{-n} A a
$$

where $\dot{a}$ and $a$ are column vectors with the elements:

$$
\dot{a}=\frac{\mathrm{d}}{\mathrm{d} t}\left(\left[N_{i}\right]-\left[N_{i}\right]^{0}\right) \text {, }
$$

and

$$
a=\left[N_{i}\right]-\left[N_{i}\right]^{0}
$$

$\left[N_{i}\right]$ and $\left[N_{i}\right]^{0}$ are the actual and the equilibrium concentrations, respectively. $A$ is a square matrix, the elements of which depend on the equilibrium properties only. ${ }^{2,6}$ Since the reaction mechanism in principle consists of an infinite number of reaction steps, the matrix has an infinite number of elements. In order to solve the eigenvalue problem, a finite number of reaction steps has to be introduced. A reasonable choice may be obtained by considering the distribution of NMA over the different polymeric species present in the solution. This distribution is given by:

$$
\phi(i)=\frac{\left[N_{i}\right]}{\sum_{i}\left[N_{i}\right]}
$$

or

and

$$
\phi(1)=\frac{\left[N_{1}\right]}{\sum_{j}\left[N_{j}\right]}
$$

$$
\phi(i)=\frac{K_{1}}{K_{n}^{2} \sum_{j}\left[N_{j}\right]}\left(K_{n}\left[N_{1}\right]\right)^{i} \text { for } i \geq 2
$$

Acta Chem. Scand. 24 (1970) No. 7 
where $\phi(i)$ is the fraction of the total number of chains which exists as $i$-mers. The value of $n$ used is the smallest number for which

$$
\sum_{i=1}^{n} \phi(i)>0.9
$$

This means that less than $10 \%$ of the chains present exist as polymers with more than $n$ units. The equilibrium constants used are $K_{1}=1 \mathbf{M}^{-1}$ and $K_{n}=45 \mathrm{M}^{-1}$. These numbers are obtained by extrapolating the values of the equilibrium constants at 25,35 , and $50^{\circ} \mathrm{C}$ reported in the literature to $20^{\circ} \mathrm{C} .^{3}$ The eigenvalue problem of the matrix is solved for different overall concentrations of NMA, $C_{1}$. Since the value of $K_{1}$ is rather uncertainly determined the eigenvalue problem is solved also for $K_{1}=5 \mathrm{M}^{-1}$. The order of the matrix used together with three of the resulting eigenvalues are given in Table 1.

Table 1. Three eigenvalues and the size of the matrix used. Equilibrium constants used are $K_{1}=5$ and $K_{n}=45 \mathrm{M}^{-1}$.

\begin{tabular}{ccccc}
\hline$C_{1}, \mathrm{M}$ & $\left|\lambda_{1}\right|$ & $\left|\lambda_{2}\right|$ & $\left|\lambda_{n}\right|$ & $n$ \\
\hline & & & & \\
0.0250 & 2.21 & 0.81 & 0.05 & 3 \\
0.0449 & 3.09 & 2.13 & 0.03 & 5 \\
0.0599 & 3.30 & 2.55 & 0.02 & 6 \\
0.0851 & 3.49 & 3.05 & 0.01 & 8 \\
0.1153 & 3.60 & 3.32 & 0.01 & 10 \\
0.2298 & 3.75 & 3.64 & 0.003 & 16 \\
\hline
\end{tabular}

If a small amount of DMA is added to a solution of NMA in carbon tetrachloride the relaxation parameters would be expected to change. This change can be related either to the fact that the DMA molecules are proton acceptors and therefore might take part in the reaction mechanism, or to the fact that the presence of DMA might change the thermodynamic properties of the solution. A simple way of describing the reaction between DMA and NMA would be:

$$
\begin{gathered}
N_{1}+N_{1} \rightleftharpoons N_{2} \\
D+N_{1} \rightleftharpoons N_{1} D \\
N_{1}+N_{2} \rightleftharpoons N_{3} \\
D+N_{2} \rightleftharpoons N_{2} D \\
\cdot \\
\cdot \\
\cdot \\
N_{1}+N_{i} \rightleftharpoons N_{i+1} \\
D+N_{i} \rightleftharpoons N_{i} D
\end{gathered}
$$


Since the hydrogen bond between two molecules of NMA is similar to that between a molecule of NMA and one of DMA, and since the probability of forming a bond between two NMA molecules by collision is twice that of forming a bond between a molecule of NMA and one of DMA, it is reasonable to introduce:

$$
\begin{aligned}
& K_{1}{ }^{\prime}=\frac{\left[N_{1} D\right]}{\left[N_{1}\right][D]}=\frac{K_{1}}{2} \\
& K_{n}{ }^{\prime}=\frac{\left[N_{i} D\right]}{\left[N_{i}\right][D]}=\frac{K_{n}}{2} \text { for } i \geq 2
\end{aligned}
$$

The overall concentration of NMA, $C_{1}$, and of DMA, $C_{2}$, in the mixture is given by:

$$
C_{1}=\sum_{i=1}^{n}\left(i\left[N_{i}\right]+i\left[N_{i} D\right]\right)
$$

and

$$
C_{2}=[D]+\sum_{i=1}^{n}\left[N_{i} D\right]
$$

or

$$
C_{1}=\left(1+K_{n}{ }^{\prime}[D]\right)\left[1+K_{1}\left[N_{1}\right] \frac{2-K_{n}\left[N_{1}\right]}{\left(1-K_{n}\left[N_{1}\right)^{2}\right.}\right]\left[N_{1}\right]+\left(K_{1}{ }^{\prime}-K_{n}{ }^{\prime}\right)\left[N_{1}\right][D]
$$

and

$$
C_{2}=[D]\left[1+K_{1}^{\prime}\left[N_{1}\right]+\frac{K_{1} K_{n}{ }^{\prime}\left[N_{1}\right]^{2}}{1-K_{n}\left[N_{1}\right]}\right]
$$

From eqn. (19) and eqn. (20) it is possible to calculate the amount of DMA which associates with NMA. This calculation shows that more than $90 \%$ of the DMA added exists as monomer in the solutions considered. Consequently a change in the relaxation parameters due to an interaction between NMA and DMA molecules is expected to be small. Since a considerably change is observed, it must be attributed to the effect of DMA on the thermodynamic properties of the system.

\section{EXPERIMENTAL}

The NMA used was fractionally recrystallized at room temperature. The NMA as well as the carbon tetrachloride was dried by passing through a column of molecular sieve (Linde $4 \mathrm{~A}$ ). The sound absorption coefficients of the solutions were measured at $20.0 \pm 0.1^{\circ} \mathrm{C}$ in the frequency range $10-300 \mathrm{Mc}$ by means of a pulse technique using a cell designed for very hygroscopic solutions. The sound absorption coefficient of the pure carbon tetrachloride divided by the frequency squared was found to be independent of the frequency in the frequency range $10-170 \mathrm{Mc}$. At higher frequencies a slight decrease in $\alpha / v^{2}$ is observed. This decrease was taken into account when the absorption of the solutions was calculated. From the absorption data and eqn. (1) the best theoretical curves and relaxation frequencies are calculated using an iterative least squares method. 


\section{RESULTS}

The frequency dependence of $\left(\alpha / \nu^{2}\right)-B$ for four different concentrations of NMA in carbon tetrachloride is shown in Fig. la and for four concentrations of NMA in DMA/CCl 4 in Fig. 1b. The observed relaxation is described by eqn. (1), this means by one relaxation time only. The goodness of fit parameter, $F^{5},{ }^{5}$ and the values of $\tau_{0} ; B$, and $A$ at the different concentrations are given in Table 2.

Fig. 2 shows the graph of the inverse of the observed relaxation time squared, $\left(1 / \tau_{0}\right)^{2}$, plotted vs, the overall concentration of NMA, $C_{1}$.
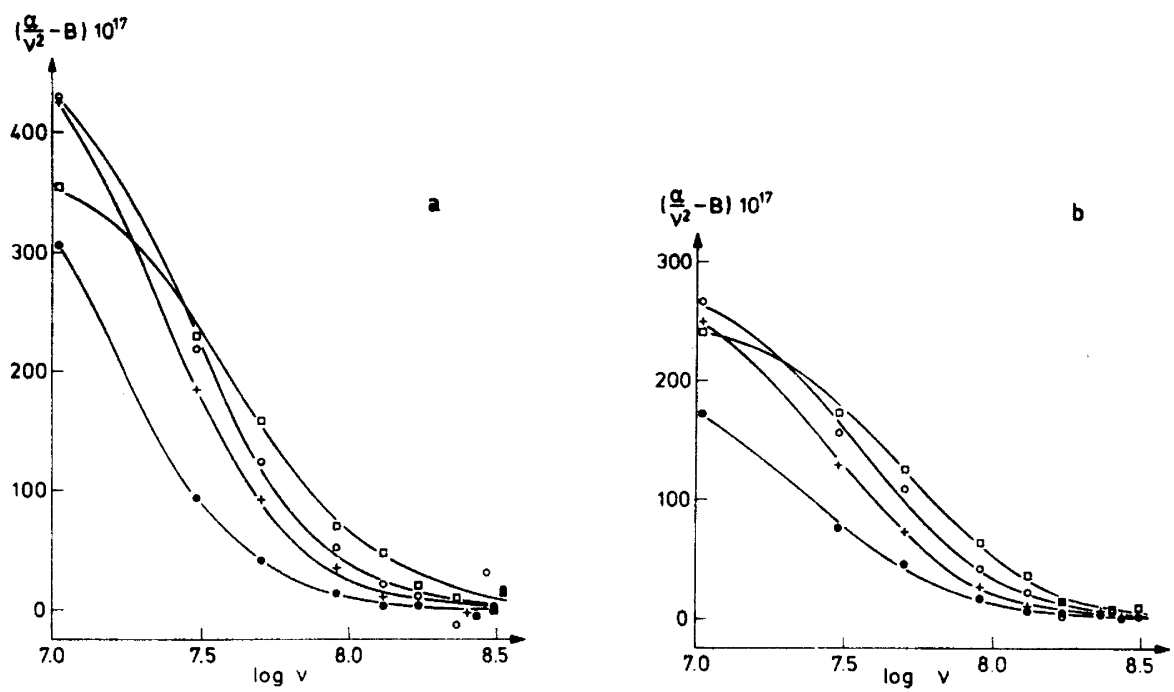

Fig. 1. The sound absorption coefficient divided by the frequency squared plotted $v 8$. the logarithm of the frequency. The curves drawn are the best theoretical curves obtained by fitting the data to eqn. (1). a: NMA dissolved in $\mathrm{CCl}_{4}$. $0.0406 \mathrm{M},+: 0.0801 \mathrm{M}$, $0: 0.1189 \mathrm{M}, \square: 0.2316 \mathrm{M}$. b: NMA dissolved in DMA/CCl, mixtures which contain $0.0310 \mathrm{M}$ DMA. : $0.0448 \mathrm{M}$, + : $0.0751 \mathrm{M}, \mathrm{O}: 0.1198 \mathrm{M}, \square: 0.2082 \mathrm{M}$.

\section{DISCUSSION}

Fig. 2 shows a straight line with a positive intercept in the concentration range 0.02 to $0.1 \mathrm{M}$. If the observed relaxation is attributed to the $\mathrm{H}$-bond association of solute molecules, as has frequently been done for relaxations occurring in the megacycle region, ${ }^{1,7,9}$ the ultrasonic data in the mentioned concentration range can be interpreted in terms of a monomer-dimer mechanism. However, this model does not apply to the infrared data, nor does it agree with the general viewpoint of amide association. The question then arises whether or not the experimental results can be explained in terms of a polymerization model. As recently pointed out ${ }^{2}$ a multistep mechanism 
Table 2. Ultrasonic relaxation parameters and goodness of fit parameter, $F$, resulting from fitting the data to eqn. (1). Results of solutions containing 0.031 M DMA are given in parentheses.

\begin{tabular}{|c|c|c|c|c|}
\hline$C_{1}, \mathrm{M}$ & $\begin{array}{c}A \times 10^{17} \\
\sec ^{2} \mathrm{~cm}^{-1}\end{array}$ & $\begin{array}{c}B \times 10^{17} \\
\mathrm{sec}^{2} \mathrm{~cm}^{-1}\end{array}$ & $\begin{array}{c}\tau_{0} \times 10^{\circ} \\
\sec \end{array}$ & $F$ \\
\hline $\begin{array}{c}0.0406 \\
(0.0448)\end{array}$ & $\begin{array}{c}447 \\
(203)\end{array}$ & $\begin{array}{c}528 \\
(510)\end{array}$ & $\begin{array}{l}10.01 \\
(6.46)\end{array}$ & $\begin{array}{c}0.3 \\
(0.3)\end{array}$ \\
\hline 0.0640 & 527 & 521 & 7.91 & 0.6 \\
\hline $\begin{array}{c}0.0801 \\
(0.0751)\end{array}$ & $\begin{array}{c}514 \\
(283)\end{array}$ & $\begin{array}{c}516 \\
(501)\end{array}$ & $\begin{array}{c}6.96 \\
(5.57)\end{array}$ & $\begin{array}{c}0.4 \\
(0.3)\end{array}$ \\
\hline $\begin{array}{l}0.0813 \\
0.1123\end{array}$ & $\begin{array}{l}540 \\
518\end{array}$ & $\begin{array}{l}515 \\
506\end{array}$ & $\begin{array}{l}7.14 \\
5.92\end{array}$ & $\begin{array}{c}0.5 \\
0.8\end{array}$ \\
\hline $\begin{array}{c}0.1189 \\
(0.1198)\end{array}$ & $\begin{array}{c}486 \\
(287)\end{array}$ & $\begin{array}{c}504 \\
(486)\end{array}$ & $\begin{array}{c}5.60 \\
(4.45)\end{array}$ & $\begin{array}{c}0.8 \\
(0.7)\end{array}$ \\
\hline $\begin{array}{c}0.2316 \\
(0.2082)\end{array}$ & $\begin{array}{c}372 \\
(247)\end{array}$ & $\begin{array}{c}470 \\
(461)\end{array}$ & $\begin{array}{c}3.82 \\
(3.14)\end{array}$ & $\begin{array}{c}0.8 \\
(0.5)\end{array}$ \\
\hline
\end{tabular}

might give a single, observed relaxation if the eigenvalues of the coefficient matrix are approximately equal, or if the amplitude factor of a particular relaxation time dominates. The former approximation is not fulfilled here while the latter gives

$$
1 / \tau_{0}=k_{-n}\left|\lambda_{i}\right|
$$

$\lambda_{j}$ is a particular eigenvalue. Since the experimental results show that no relaxation occurs at higher frequencies, we take $j=1$. From eqn. (21) it is seen

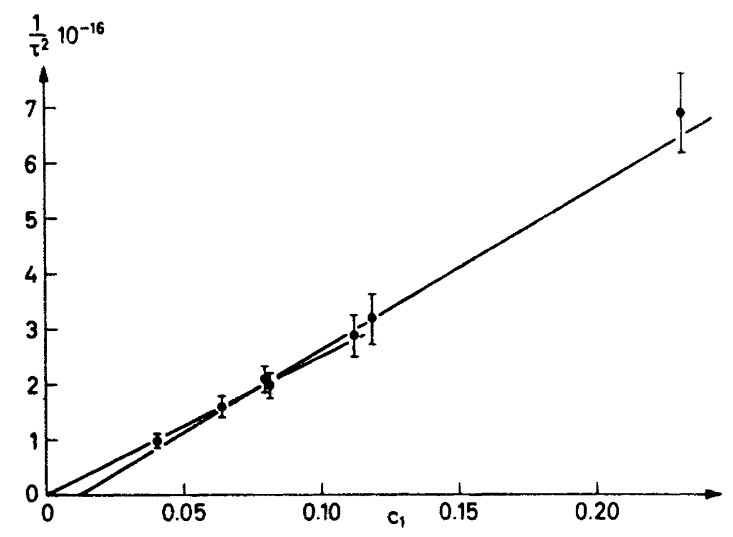

Fig. 2. The reciprocal relaxation time squared plotted vs. the overall concentration of NMA for the NMA/CCl, mixtures.

Acta Chem. Scand. 24 (1970) No. ? 
that the proportionality factor between $1 / \tau_{0}$ and $\left|\lambda_{1}\right|$ is $k_{-n}$. Fig. 3 shows $1 / \tau_{0}$ and $k_{-n}\left|\lambda_{1}\right|$ plotted $v s$. the overall concentration of $\mathrm{NMA}$.

The rate constants obtained are given in Table 3 . It is seen that in the concentration range 0.02 to $0.1 \mathrm{M}$ the two curves agree within experimental

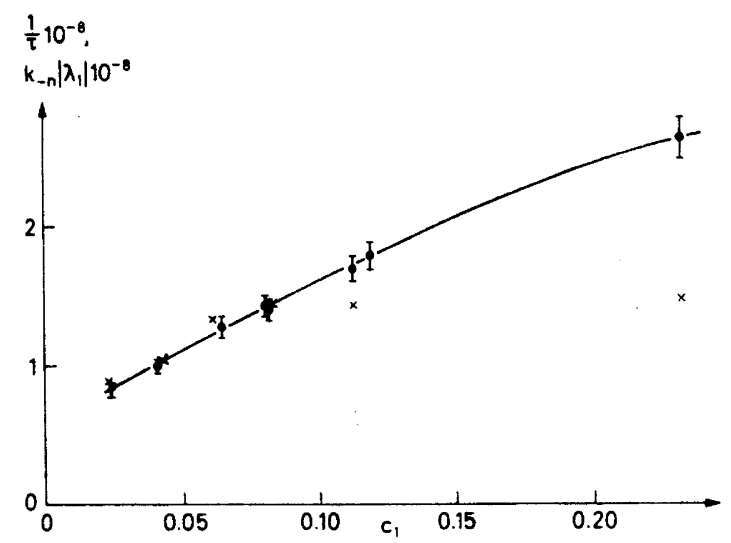

Fig. 3. The dependence of the reciprocal relaxation time and of $k_{-n}\left|\lambda_{1}\right|$ on the overall concentration of NMA for the $\mathrm{NMA} / \mathrm{CCl}_{4}$ mixtures.

Table 3. Values of the rate constants obtained using $K_{1}=1 \mathrm{M}^{-1}$ and $5 \mathrm{M}^{-1}$, and $K_{n}=45 \mathrm{M}^{-1}$.

\begin{tabular}{ccc}
\hline$k_{1}, \mathrm{M}^{-1} \mathrm{sec}^{-1}$ & $k_{n}, \mathrm{M}^{-1} \mathrm{sec}^{-1}$ & $k_{-n}, \mathrm{sec}^{-1}$ \\
\hline $3.8 \times 10^{7}-1.9 \times 10^{8}$ & $1.7 \times 10^{\circ}$ & $3.8 \times 10^{7}$ \\
\hline
\end{tabular}

error. Above 0.1 M, however, the two curves diverge, raising the possibility that an alternative mechanism must be considered. It should be noted that although a plot of $1 / \tau_{0}^{2} v s . C_{1}$ shows a straight line in the extended concentration range, the monomer-dimer mechanism could not now explain the results since this line has a negative intercept. A possible alternative reaction which might explain the observed relaxation is the internal rotation about the $\mathrm{C}-\mathrm{N}$ bond in NMA, but this mechanism seems unlikely in view of the dependence of $1 / \tau_{0}$ on $C_{1}$ in the low concentration range.

The use of the concentration dependence of $1 / \tau_{0}$ to evaluate rate constants involves the basic assumption that the solute used does not affect the thermodynamic properties of the solution over the concentration range studied. A decrease in the relaxation strength, $A$, with concentration might cause an increase in the fitted value of $1 / \tau_{0}$. Fig. 4 shows the graph of $A$ vs. $C_{1}$ for the systems investigated.

It appears that the concentration dependence of $A$ shows a distinct maximum, and that the addition of DMA causes a parallel shift of the curve to lower values of $A$. In addition, it can be calculated from Table 1 that the value of 


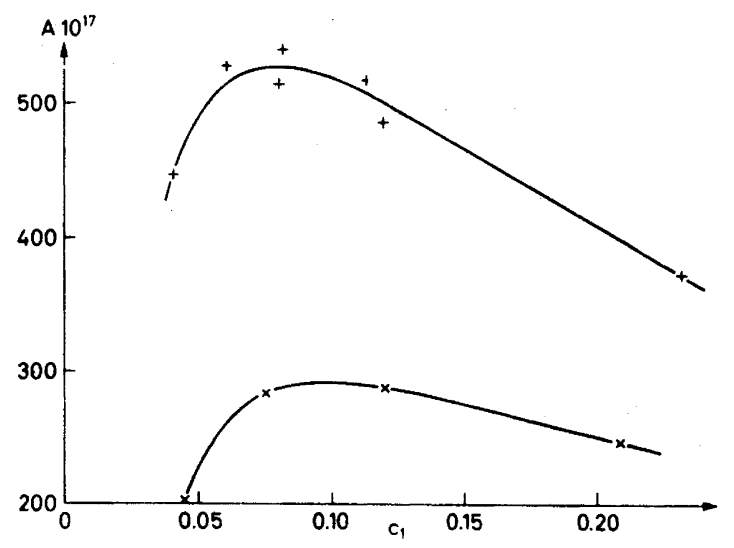

Fig. 4. The relaxation strength plotted $v s$. the overall concentration of NMA. +: NMA/CCl, solutions, $x:$ NMADMA/CCl, solutions.

$1 / \tau_{0}$ of an $\mathrm{NMA} / \mathrm{CCl}_{4}$ solution is increased by a constant factor of 1.3 by the addition of $0.031 \mathrm{M}$ DMA, throughout the entire concentration range of NMA. These two observations together with the previous conclusion that a small amount of DMA has a negligible effect on the association of NMA indicate that the change in $A$ and $\tau_{0}$ upon the addition of DMA is caused primarily by a change in the thermodynamic properties of the system. Since the DMA affects the relaxation strength so drastically, there is no reason to expect the thermodynamic properties of the solution to be independent of $C_{1}$ throughout the concentration range used. The observed decrease in $A$ at high NMA concentrations is explained partly on this basis. This decrease causes the fitted values of $1 / \tau_{0}$ in this concentration range to be higher than expected from the kinetic mechanism alone.

\section{CONCLUSION}

The relaxation spectra of NMA dissolved in carbon tetrachloride can be explained by the generally accepted $\mathrm{H}$-bond polymerization model in the concentration range 0.02 to $0.1 \mathrm{M}$. By assuming the association rate constants to be the same for all steps beyond the first, and by assuming all dissociation rate constants to be equal, it is possible to combine the ultrasonic results with equilibrium results determined by infrared spectroscopy and to calculate approximate values of the rate constants involved. The fact that the relaxation data can also be fitted to the simpler monomer-dimer mechanism illustrates how careful it is necessary to be when interpreting ultrasonic data. Internal rotation about the $\mathrm{C}-\mathrm{N}$ bond in $\mathrm{NMA}$ is ruled out as a cause of the observed relaxation because of the dependence of $1 / \tau_{0}$ on concentration in the low concentration range.

The increase in $1 / \tau_{0}$ for NMA concentrations above $0.1 \mathrm{M}$ is attributed primarily to the decrease in the relaxation strength, $A$, in this concentration

Acta Chem. Scand. 24 (1970) No. 7 
range, which is due to the effect of NMA on the thermodynamic properties on which $A$ depends. The fact that the addition of a small amount of DMA to the NMA $/ \mathrm{CCl}_{4}$ solutions decreases the value of $A$ drastically while at the same time $1 / \tau_{0}$ increases supports this point of view.

Acknowledgement. The authors are deeply indebted to Professor Thor A. Bak for his constant interest in this work.

\section{REFERENCES}

1. Rassing, J. and Østerberg, O. Acta Chem. Scand. 23 (1969) 693.

2. Rassing, J. Proc. 1st Intern. Conf. Calorimetry and Thermodynamics, Warsaw 1969.

3. Lowenstein, H., Lassen, H. and Hvidt, Aa. Acta Chem. Scand. 24 (1970) 1887.

4. Klotz, I. M. and Franzen, J. S. J. Am. Chem. Soc. 84 (1962) 3461.

5. Rassing, J. and Lassen, H. Acta Chem. Scand. 23 (1969) 1007.

6. Rassing, J. and Jensen, B. N. Acta Chem. Scand. 24 (1970) 855.

7. Rassing, J., Østerberg, O. and Bak, T. A. Acta Chem. Scand. 21 (1967) 1443.

8. Hammes, G. G. Accounts Chem. Res. 1 (1968) 321.

Received January 15, 1970. 